\title{
Spinal fusion for paediatric lumbosacral spondylolisthesis
}

\author{
Horn $\mathrm{A}^{1}$, Watt $\mathrm{JP}^{2}$, Dunn $\mathrm{RN}^{3}$
}

1 MBChB(UP), MMed Orth(UCT), FC Orth(SA); Department of Orthopaedic Surgery, University of Cape Town and Red Cross War Memorial Children's Hospital, Cape Town, South Africa

2 MBChB(UP), MMed Orth(Stel), FC Orth(SA); AOSpine fellow, Department of Orthopaedic Surgery, Groote Schuur Hospital, Cape Town, South Africa

3 MBChB(UCT), MMed Orth(UCT), FC Orth(SA); HOD/Pieter Moll and Nuffield Chair of Orthopaedic Surgery, University of Cape Town,

Groote Schuur Hospital and Red Cross Children's Hospital, Cape Town, South Africa

Corresponding author: Dr Anria Horn, Postnet suite 342, Private Bag X18, Rondebosch, Cape Town, South Africa; tel: +27 (0)21 4045108 ; fax: +27 (0)21 447 2709; email: anriahorn@gmail.com

\begin{abstract}
Background: Paediatric spondylolisthesis can range from a benign non-progressive asymptomatic condition to rapidly progressive slips with associated debilitating axial and radicular pain. We reviewed our experience as to the presentation, surgical management and outcomes following surgery of this relatively rare condition.

Methods: We identified 24 paediatric patients who underwent surgery for spondylolisthesis from 2006-2017. The cohort consisted of 17 females and seven males with a median age of 13 years (IQR 11-14) at the time of surgery. A case note and imaging review was conducted. Presenting history and examination, aetiology and degree of slip, surgical technique employed, complications and outcome were analysed.

Results: Most patients presented with a combination of lower back and radicular symptoms, with five having radiculopathy as a single complaint. Seventeen cases involved instrumentation and 18 interbody fusions, with interbody cages used in 14, and bone graft alone in four. All but the four uninstrumented interbody fusions were performed with an all-posterior approach. In 11 of grade 1 and 2 slip cases, the listhesis was completely reduced. Four patients were partially reduced and in six patients, including one spondyloptosis, fusion was in situ. All patients with radicular symptoms were decompressed surgically, along with those where the listhesis was reduced. All but one patient has complete resolution of presenting symptoms at latest follow-up. Four patients required re-operation for complications related to the surgery.

Conclusion: Paediatric spondylolisthesis can be successfully managed employing a variety of techniques. For low grade slips, reduction and interbody fusion is a safe and reliable method of improving spinal sagittal balance and relieving symptoms related to nerve root compression and spinal instability. For higher grade slips, reduction was associated with instrumentation failure in two patients and in-situ fusion via a combined anterior and posterior approach had a better outcome in our patients.
\end{abstract}

Level of evidence: Level 4 - Case series

Key words: spinal fusion, paediatrics, spondylolisthesis, spine deformity

Citation: Horn A, Watt JP, Dunn RN. Spinal fusion for paediatric lumbosacral spondylolisthesis. SA Orthop J 2018;17(4):39-43.

http://dx.doi.org/10.17159/2309-8309/2018/v17n4a5

Editor: Dr J Davis, Stellenbosch University

Received: February 2018

Accepted: June 2018

Published: November 2018

Copyright: $\odot 2018$ Horn A. This is an open-access article distributed under the terms of the Creative Commons Attribution Licence, which permits unrestricted use, distribution and reproduction in any medium, provided the original author and source are credited.

Funding: No external funding was required for the conduction of this research.

Conflict of interest: None of the authors have any conflicts of interest to declare. 


\section{Introduction}

Paediatric lumbosacral spondylolisthesis remains a challenging condition for the spinal surgeon to treat. Low grade slips $(<25 \%)^{1}$ are often asymptomatic, follow a benign clinical course and are as such either undiagnosed or diagnosed incidentally. When symptomatic, the presenting complaint is usually radicular limb pain from foraminal stenosis, particularly in the lytic group. Higher grade slips are frequently progressive and present with disabling pain, neurological symptoms, abnormal posture or a combination of these. Indications for surgery and surgical techniques vary widely, and the superiority of one technique over another has not been conclusively proven in the literature. ${ }^{2,3}$ This is not unexpected in such a heterogenous patient population.

Historically uninstrumented fusions, either anterior, posterior or both, were the mainstay of treatment., ${ }^{4,5}$ This was complemented by prolonged casting or bracing and led to satisfactory outcomes except for a relatively high rate of asymptomatic pseudarthrosis formation and concerns regarding slip progression. Deformity correction can however not be fully addressed using this approach.

With more advanced spinal instrumentation, instrumented posterior fusion in addition to anterior interbody fusion became increasingly utilised. This approach led to improved radiological parameters by allowing a degree of reduction. Union rates also improved. ${ }^{6}$ With the development of posterior-based interbody techniques and cage technology, decompression, reduction and interbody fusion became possible through a single posterior approach. This approach reduces surgical time and morbidity but may not be safe or appropriate for higher grade slips and spondyloptosis. ${ }^{7}$

The purpose of this study is to evaluate the results of operative treatment for paediatric lumbosacral spondylolisthesis at a tertiary centre in South Africa. All patients were operated on by a single surgeon. The variety of techniques employed reflects the learning curve of the surgeon, the advances and availability of instrumentation and increasing understanding of the pathomechanics of this condition.

\section{Methods}

We performed a retrospective review of a prospectively maintained single surgeon database and identified 24 paediatric patients that underwent listhesis surgery during a nine-year period (2006-2015) both at the Red Cross Children's Hospital and in private practice. The case records and radiology for these patients were reviewed and data captured regarding demographic details, presenting complaint, clinical and radiological findings and surgery performed. Segmental lordosis was measured using the angle subtended by the superior edge of the sacrum and the superior edge of L5 vertebra using a variety of radiological software.

Outcome parameters captured were time to radiological union, residual lumbar kyphosis and resolution of symptoms. Radiological union was defined as a well-consolidated fusion mass with cross trabeculation on X-ray and no evidence of progression of the slip or failure of instrumentation.

Due to the relatively small number of heterogenous cases, only descriptive statistics were used.

\section{Surgical technique}

\section{Posterior only decompression and transpedicular lumbar interbody fusion (TLIF)}

The patient was positioned prone and the spine exposed in the standard fashion. Pedicle screws were inserted in the levels adjacent to the listhesis. If available, long-eared reduction screws were placed in the cephalad vertebrae to facilitate rod connection and subsequent reduction. In the case of a lytic pathology, which was the usual indication for this procedure, the rattle/Gill fragment (loose posterior arch) was removed. It was morselised for graft. The exiting roots were identified medial to the pedicle and followed out debriding all the compressive tissue from the pars defect/lysis. An annulotomy was performed and the disc removed to visualise the endplates and anterior annulus. On occasion a bilateral annulotomy was performed to improve disc clearance and release. The TLIF PEEK (polyetheretherketone) cage was then inserted as anterior as possible on the inferior vertebra's endplate prior to rod insertion, while the posterior disc space was still easily accessible. The rods were then applied with slow reduction, palpating the exiting roots for tension and watching for signs of screw pull out. Once reduced, compression was applied from cephalad to caudal screws to induce segmental lordosis. Reduction was limited by concern regarding screw purchase and root tension. Complete reduction was not attempted in the higher-grade slips.

\section{Uninstrumented circumferential fusion through a combined anterior and posterior approach}

Patients were positioned supine with a bolster underneath the lumbar spine to provide hyperextension. Through a retroperitoneal approach, between the bifurcation of the great vessels, the L5/S1 disc was removed and femoral ring allograft inserted, providing some reduction of the kyphosis. Following this, the patient was re-positioned prone, and an uninstrumented posterolateral fusion performed via the Wiltse muscle-sparing approach. These patients were immobilised in a lumbosacral orthosis (LSO) incorporating a thigh. The orthosis was constructed with a bilateral articulation so that the thigh extension could be alternated every two weeks to reduce the chance of hip chondrolysis. At six weeks the leg extension was removed, and the LSO worn until three months postoperatively.

Table I: Details of 24 patients with paediatric spondylolisthesis

\begin{tabular}{|c|c|}
\hline Patients (n) & 24 \\
\hline Females (\%) & $17(71)$ \\
\hline Age at presentation in years (IQR) & $13(11,14)$ \\
\hline \multicolumn{2}{|l|}{ Type (Wiltse) } \\
\hline Type 1 - dysplastic & 5 \\
\hline Type 2 - isthmic & 19 \\
\hline \multicolumn{2}{|l|}{ Grade (Meyerding) } \\
\hline $1<25 \%$ & 8 \\
\hline 2 25\%-50\% & 9 \\
\hline $350 \%-75 \%$ & 4 \\
\hline $475 \%-100 \%$ & 2 \\
\hline Spondyloptosis & 1 \\
\hline \multicolumn{2}{|l|}{ Affected level } \\
\hline L5-S1 & 22 \\
\hline L4-L5 & 1 \\
\hline L4-S1 & 1 \\
\hline \multicolumn{2}{|l|}{ Presenting complaint } \\
\hline Lower back pain & 4 \\
\hline Radiculopathy & 5 \\
\hline Both & 15 \\
\hline Lumbar lordosis (IQR) & $9^{\circ}\left(0^{\circ}, 16^{\circ}\right)$ \\
\hline
\end{tabular}

$I Q R=$ interquartile range 


\section{Results}

The patients' demographic details, listhesis type and grade as well as presenting symptoms are summarised in Table $I$. The majority of our patients had low grade $(<50 \%)$ isthmic slips and presented with

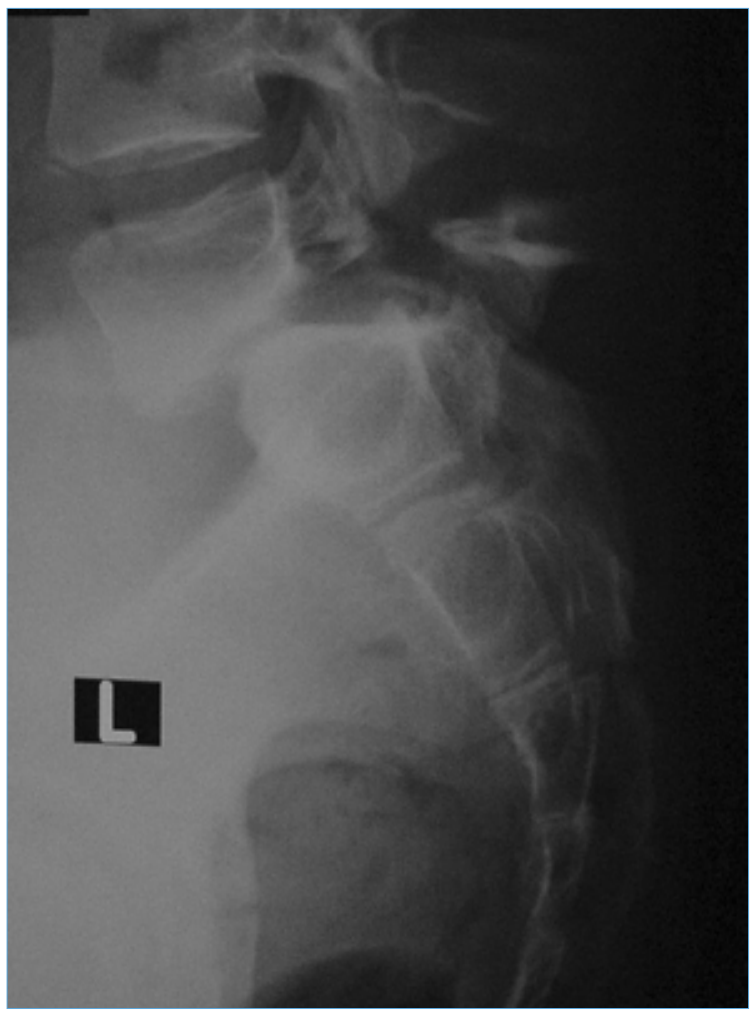

Figure 1a. Eleven-year-old gymnast with grade 2 lytic listhesis L5/S1

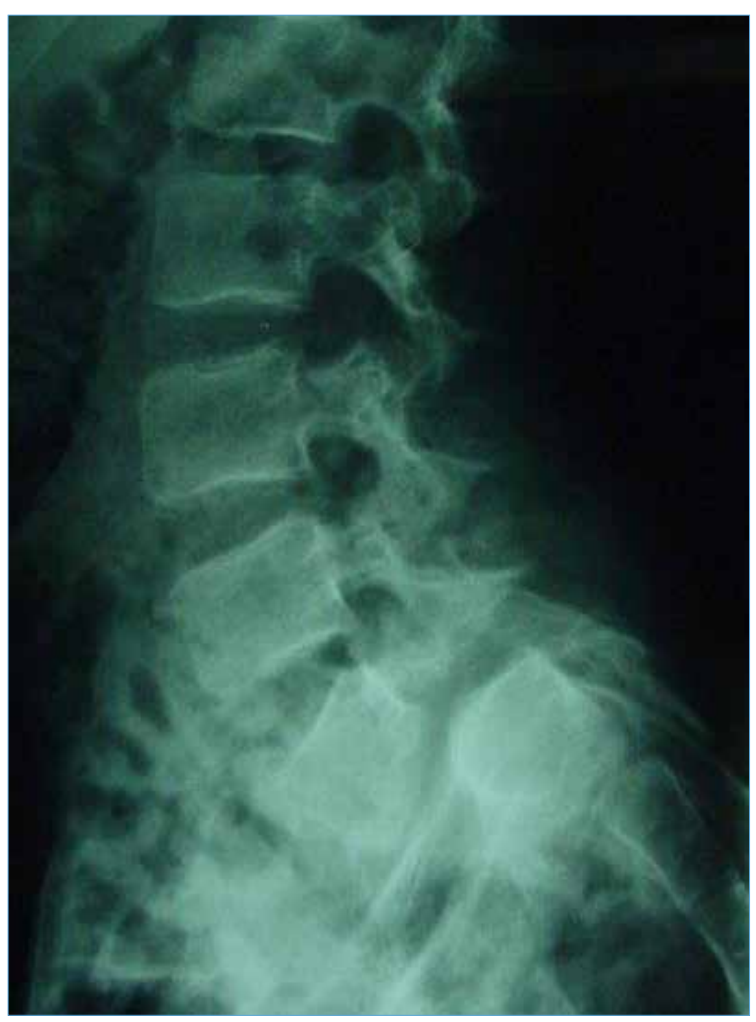

Figure 2a. Ten-year old female with grade 4 dysplastic listhesis L5/S1 a combination of lower back pain (LBP) and radicular symptoms. The radicular symptoms mostly consisted of radicular pain in the L5 and S1 distributions. Two patients had loss of motor power and one patient had urinary incontinence at presentation. This patient had a tethered cord that had been surgically released the previous year.

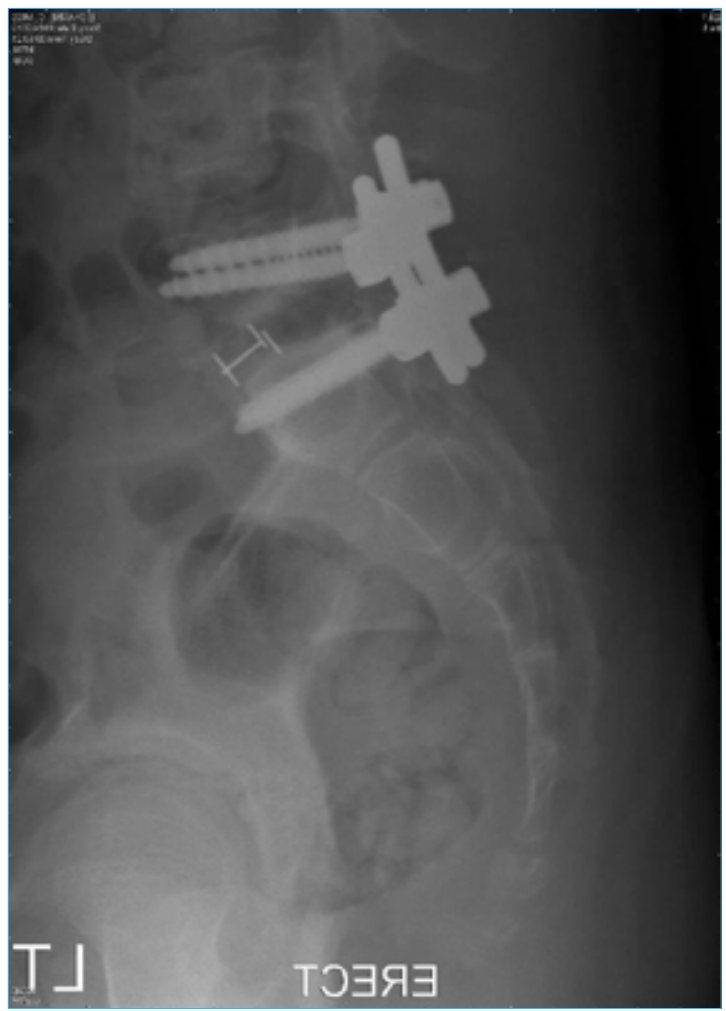

Figure 1b. Three months post-operatively. The listhesis has been reduced employing the posterior only decompression and TLIF technique

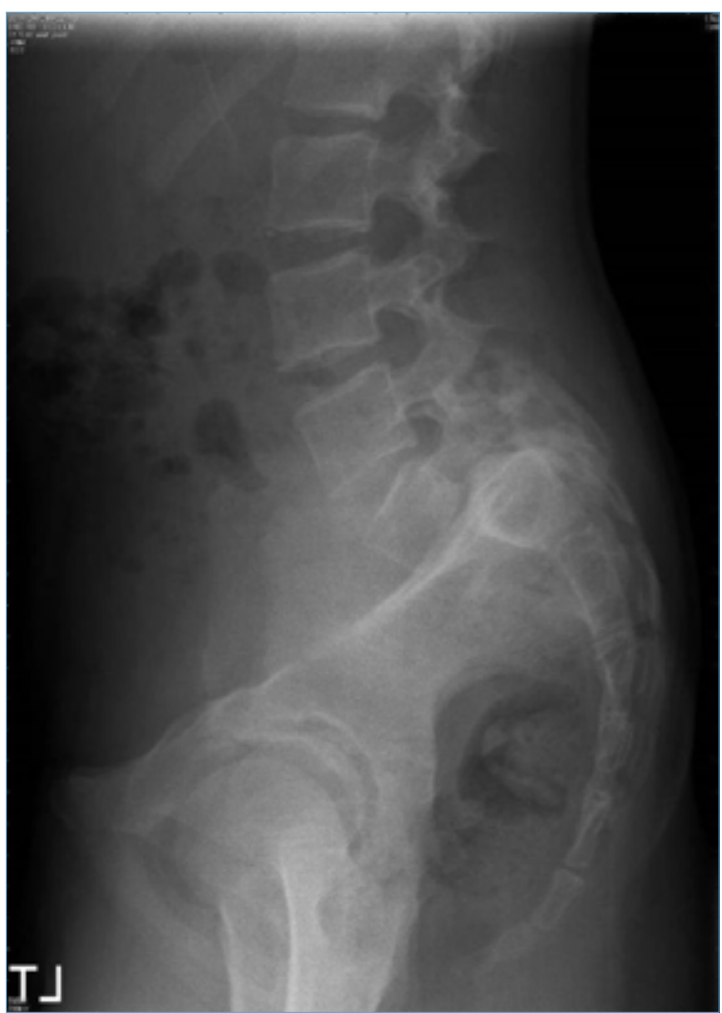

Figure $\mathbf{2 b}$. One year post-operatively, the patient has been fused in situ using a combined anterior and posterior approach. She is asymptomatic and has returned to competitive dancing. 
Table II: Details and results of surgery for the two most frequently performed procedures in our cohort

\begin{tabular}{|l|c|c|}
\hline & Posterior only decompression and TLIF & Anterior and posterior uninstrumented fusion \\
\hline Patients $(\mathrm{n})$ & 16 & 4 \\
\hline Pre-operative lumbar lordosis (IQR) & $9^{\circ}\left(0^{\circ}, 16^{\circ}\right)$ & $-9^{\circ}\left(-30^{\circ}, 9^{\circ}\right)$ \\
\hline Post-operative lumbar lordosis (IQR) & $21^{\circ}\left(12^{\circ}, 27.5^{\circ}\right)$ & $1.5^{\circ}\left(-6^{\circ}, 9^{\circ}\right)$ \\
\hline Correction of deformity & $11^{\circ}$ & $11^{\circ}$ \\
\hline Surgical time (IQR) & $135 \mathrm{~min}(110,150)$ & $182 \mathrm{~min}(145,260)$ \\
\hline Blood loss (IQR) & $300 \mathrm{ml}(250,500)$ & $193 \mathrm{ml}(150,220)$ \\
\hline Time to fusion (IQR) & 6 months $(3,15)$ & $7.5 \mathrm{months}(3,12)$
\end{tabular}

$I Q R=$ interquartile range

Associated conditions included scoliosis in two patients, and Marfan syndrome, a lumbarised $\mathrm{S} 1$ and a tethered cord in one patient each.

Fourteen of the patients participated in a wide variety of competitive sports including, but not limited to, sports associated with repetitive hyperextension of the spine such as cricket (bowling) and gymnastics. All these patients had an isthmic spondylolisthesis.

Early in the study period, posterior only decompression and instrumented fusion with pedicle screws was performed in two patients with grade 2 isthmic slips. Concerns regarding inability to improve segmental kyphosis and increase foraminal height other than with kyphogenic distraction, led to this technique being abandoned in favour of posterior only decompression and interbody fusion with the TLIF technique (see Methods section) for lower grade slips. Sixteen patients underwent this procedure (Figures 1a and b).

Two patients with grade 1 slips underwent uninstrumented posterolateral fusion early in the study period and had good clinical outcome.

Uninstrumented anterior interbody fusion combined with posterior un-instrumented fusion was performed in four patients with high grade slips throughout the study period (Figures $2 a$ and b).

The pre- and post-operative radiographic parameters as well as the surgical blood loss and duration for the two commonest procedures are tabulated in Table II. They are not directly comparable due to the vast difference in aetiology and deformity. The antero-posterior procedure was performed for high grade slips.

Of the 16 patients that underwent posterior-only decompression and TLIF, 12 had an excellent outcome in terms of radiographic union and presenting symptom resolution. Four patients in this group experienced complications. Two patients, both of whom had high grade dysplastic slips, had loss of fixation in the early post-operative phase. In one, the complication occurred while in hospital and the instrumentation was revised to include L4 with a satisfactory result. The other patient lived remotely and was asymptomatic. The complication was only detected on routine three-month post-operative X-ray. He was initially observed, and fixation was removed at 18 months post-operatively. He remained asymptomatic despite the unreduced position. One patient, with very slight (MRC grade 4) L5 weakness pre-operatively developed worsening weakness in the L5 distribution post-operatively. Surgical exploration was performed, and the reduction decreased to reduce traction on the nerve root, yet the foot drop persists at 2 years follow-up. One patient who had a grade 2 isthmic listhesis, developed proximal junctional kyphosis above the level of the fusion, which is of uncertain clinical significance.

There were no complications in either of the uninstrumented fusion groups.

Of the two patients that had posterior-only instrumented fusion without TLIF, one had catastrophic instrumentation failure with screw fracture and slip progression. This was finally revised by extending fixation from $L 4$ to the pelvis. The patient was asymptomatic at the latest follow-up.

\section{Discussion}

The goals of treatment in spondylolisthesis are first to relieve symptoms such as radicular pain and hamstring tightness, secondly to prevent slip progression and thirdly to improve or correct the lumbosacral deformity. This can be achieved to varying degrees by employing a variety of surgical techniques.

Uninstrumented posterolateral fusion in situ has been used with satisfactory results for the treatment of all grades of slips. ${ }^{5,8}$ Concerns regarding this procedure are slip progression, a high rate of nonunion requiring revision surgery, and the inability of this procedure to address the lumbosacral kyphosis. ${ }^{4,5}$ Though believed to carry a lesser risk of neurological injury, in situ fusion was associated with the development of cauda equina syndrome in 12 patients with grade 3 and 4 slips in a series by Schoenecker et al. ${ }^{9}$ and can therefore not be assumed to be without risk. In order to address these concerns, various modifications have been introduced. Circumferential uninstrumented fusion by means of a combined anterior and posterior approach have succeeded in improving fusion rates as well as addressing the kyphosis to a minor extent. ${ }^{4}$ Posterior instrumentation has also been attempted in isolation, but in our experience, this method of fixation is inadequate to maintain the reduction and allow for an optimal fusion environment.

The addition of an anterior interbody fusion using either allograft or a fusion cage has the benefit of improving the lumbosacral kyphosis and providing a larger surface area for fusion to occur. In lower grade slips the interbody cage indirectly decompresses the stenotic foramina providing reliable relief of neurological symptoms arising from nerve root compression. In our series, neurological symptoms were improved in all patients except the one case that developed a foot drop post-op. The lumbar kyphosis was corrected by $11^{\circ}$ and fusion was achieved at a mean of 6 months.

Reduction of severe slips remains a controversial subject. ${ }^{2,4}$ By reducing the lumbosacral kyphosis, spinal sagittal balance can be improved or restored with the hope of preventing the long-term effects of sagittal imbalance. ${ }^{10}$ Some authors also postulate that by creating a more favourable biomechanical environment by means of reduction, fusion rates will improve. ${ }^{6,11}$ To the contrary, certain other authors found no difference in clinical outcomes with reduction compared to in-situ fusion. ${ }^{12}$ This risk of neurological damage was believed to be enhanced by attempting reduction of the listhesis, ${ }^{4,7}$ but has been shown to be safe in more recent publications. $^{3}$ In our experience, reduction is safe in lower grade slips when performing posterior only decompression and TLIF.

The risk of neurological damage is low in low grade slips and was seen in only one of our patients who had a grade 2 slip. Attempted reduction led to instrumentation failure in two of our patients who had grade 3 and 4 slips respectively, but no neurological sequelae 
were noted with reduction of severe slips. According to a recent literature review by Longo et al., there was no significant difference in the rate of neurological injury between patients that underwent reduction and those that did not. ${ }^{3}$ This study does not comment on the grade of slip in the reported articles but concludes that reduction is safe and leads to better spinal alignment and lower pseudo-arthrosis rates. In order to minimise the risk of neurological sequelae, the sacral dome can be resected and the L5 nerve roots mobilised. Extending the fusion to L4 may also prevent instrumentation failure, although sacrificing an additional motion segment should be avoided if possible.

\section{Conclusion}

Symptomatic spondylolisthesis can be managed successfully using a variety of surgical approaches. Of the 24 patients included in our study, 23 (96\%) had satisfactory outcomes at final follow-up. Four patients (17\%) required re-operation for complications and only one $(4 \%)$ remains symptomatic at an average follow-up of 22 months.

For the treatment of low grade slips $(<50 \%)$ the authors' preferred method of treatment is posterior decompression and TLIF. Uninstrumented posterolateral fusion is another good option for low grade slips but requires a prolonged period of immobilisation, rendering it a less attractive though simpler and less expensive option. For higher grade slips we found that circumferential uninstrumented fusion by means of a combined anterior and posterior approach had the best results; however, reduction and instrumented fusion is also a safe and reliable option.

\section{Ethics statement}

The study was performed following ethical approval form the University of Cape Town Human Research Ethics Committee (HREC R039/2013).

Research was performed in accordance with the Responsible Research Publication Position statements as developed at the 2nd World Conference on Research Integrity, 2010.

\section{References}

1. Meyerding H. Spondylolisthesis. Surg Gynecol Obstet. 1932;54:371-77.

2. Violas P, Lucas G. L5S1 spondylolisthesis in children and adolescents. Orthop Traumatol Surg Research. 2016;102(1):S141-S7

3. Longo UG, Loppini M, Romeo G, Maffulli N, Denaro V. Evidencebased surgical management of spondylolisthesis: reduction or arthrodesis in situ. J Bone Joint Surg Am. 2014;96(1):53-58.

4. Bradford DS, Boachie-Adjei $O$. Treatment of severe spondylolisthesis by anterior and posterior reduction and stabilization. A long-term follow-up study. J Bone Joint Surg Am. 1990;72(7):1060-66.

5. Seitsalo S, Osterman K, Hyvarinen H, Schlenzka D, Poussa M. Severe spondylolisthesis in children and adolescents. A long-term review of fusion in situ. Bone Joint J. 1990;72(2):259-65.

6. Muschik M, Zippel H, Perka C. Surgical management of severe spondylolisthesis in children and adolescents: anterior fusion in situ versus anterior spondylodesis with posterior transpedicular instrumentation and reduction. Spine. 1997;22(17):2036-42.

7. Lonstein JE. Spondylolisthesis in children: cause, natural history, and management. Spine. 1999;24(24):2640.

8. Grzegorzewski A, Kumar SJ. In situ posterolateral spine arthrodesis for grades III, IV, and V spondylolisthesis in children and adolescents. J Pediatr Orthop. 2000;20(4):506-11.

9. Schoenecker PL, Cole H, Herring J, Capelli A, Bradford D. Cauda equina syndrome after in situ arthrodesis for severe spondylolisthesis at the lumbosacral junction. $J$ Bone Joint Surg Am. 1990;72(3):369-77.
10. Labelle H, Mac-Thiong J-M, Roussouly P. Spino-pelvic sagittal balance of spondylolisthesis: a review and classification. Eur Spine J. 2011;20(5):641.

11. Rodriguez-Olaverri JC, Zimick NC, Merola A, Vicente J, Rodriguez J, Tabuenca $\mathrm{A}$, et al. Comparing the clinical and radiological outcomes of pedicular transvertebral screw fixation of the lumbosacral spine in spondylolisthesis versus unilateral transforaminal lumbar interbody fusion (TLIF) with posterior fixation using anterior cages. Spine. 2008;33(18):1977-81.

12. Poussa $M$, Remes $V$, Lamberg $T$, Tervahartiala $P$, Schlenzka $\mathrm{D}$, Yriönen $\mathrm{T}$, et al. Treatment of severe spondylolisthesis in adolescence with reduction or fusion in situ: long-term clinical, radiologic, and functional outcome. Spine. 2006;31(5):583-90. 